\title{
Membrane Anchor of Cytochrome P450 Reductase Suppresses the Uncoupling of Cytochrome P450
}

\author{
Masayoshi Miyamoto, ${ }^{a, \#}$ Taku Yamashita, ${ }^{b, \#}$ Yuki Yasuhara, ${ }^{a}$ Akinori Hayasaki, ${ }^{a}$ \\ Yukari Hosokawa, ${ }^{a}$ Hirofumi Tsujino, ${ }^{a}$ and Tadayuki Uno*,a \\ ${ }^{a}$ Graduate School of Pharmaceutical Sciences, Osaka University; Osaka 565-0871, Japan: and ${ }^{b}$ School of \\ Pharmacy and Pharmaceutical Sciences, Mukogawa Women's University; Hyogo 663-8179, Japan. \\ Received January 11, 2015; accepted January 16, 2015
}

\begin{abstract}
Cytochrome P450 reductase (CPR) is an important redox partner of microsomal CYPs. CPR is composed of a membrane anchor and a catalytic domain that contains FAD and flavin mononucleotide (FMN) as redox centers and mediates electron transfer to CYP. Although the CPR membrane anchor is believed to be requisite for interaction with $C Y P$, its physiological role is still controversial. To clarify the role of the anchor, we constructed a mutant $(\triangle 60-C P R)$ in which the $\mathrm{N}$-terminal membrane anchor was truncated, and studied its effect on binding properties, electron transfer to CYP2C19, and drug metabolism. We found that $\triangle 60-C P R$ could bind to and transfer electrons to CYP2C19 as efficiently as WT-CPR, even in the absence of lipid membrane. In accordance with this, $\triangle 60-C P R$ could mediate metabolism of amitriptyline (AMT) and imipramine (IMP) in the absence of lipids, although activity was diminished. However, $\triangle 60-C P R$ failed to metabolize omeprazole (OPZ) and lansoprazole (LPZ). To clarify the reason for this discrepancy in drug metabolism, we investigated the uncoupling reaction of the CYP catalytic cycle. By measuring the amount of $\mathrm{H}_{2} \mathrm{O}_{2}$ by-product, we found that shunt pathways were markedly activated in the presence of OPZ/LPZ in the $\triangle 60-C P R$ mutant. Because $\mathrm{H}_{2} \mathrm{O}_{2}$ levels varied among the drugs, we conclude that the proton network in the distal pocket of CYP2C19 is perturbed differently by different drugs, and activated oxygen is degraded to become $\mathrm{H}_{2} \mathrm{O}_{2}$. Therefore, we propose a novel role for the membrane anchor as a suppressor of the uncoupling reaction in drug metabolism by CYP.
\end{abstract}

Key words cytochrome P450 reductase; membrane anchor; electron transfer; drug metabolism; shunt pathway

Cytochrome $\mathrm{P} 450$ reductase (CPR) is a membrane-anchored protein equipped with two flavins (FAD and flavin mononucleotide $(\mathrm{FMN})$ ), and is capable of electron transfer to various heme proteins. ${ }^{1-5)}$ Among its redox partners, its interactions with CYPs have been intensively studied, because CYPs are involved in the metabolism of biologically important substances, such as steroids, fatty acids, and vitamin D, as well as xenobiotics. Human CYP is reported to have 57 isoforms, and CYP1A2, 2C9, 2C19, 2D6, and 3A4 are the main isoforms involved in drug metabolism in humans. ${ }^{6,7)}$ Interestingly, these CYPs share the same CPR as a redox partner, indicating the existence of a common mechanism in electron transfer events.

Human CPR exists in the membrane of the endoplasmic reticulum. Its amino acid sequence is $c a$. $90 \%$ identical to that of other mammalian CPRs. ${ }^{8-10}$ The crystal structures of mammalian CPRs were recently reported, ${ }^{11,12)}$ and suggested that CPRs consist of one N-terminal membrane anchor domain and three hydrophilic domains: FAD-binding, connecting, and FMN-binding domains. ${ }^{11)}$ The two flavins constitute the redox center of CPR, and FAD receives a hydride anion from exogenous reduced nicotinamide adenine dinucleotide phosphate (NADPH) to transfer electrons to FMN. The connecting domain links the FMN- and FAD-binding domains, and the FMN-binding domain is believed to be involved in the interaction with CYP. The surface of the FMN-binding domain is negatively charged with acidic residues, while basic residues are localized to the proximal surface of CYPs. ${ }^{13-18)}$ These residues contribute electrostatically to the formation

\footnotetext{
\# These authors contributed equally to this work.

of the CPR-CYP complex, facilitating electron transfer from FMN to the heme in CYP. Surprisingly, the charged surface of the FMN-binding domain was not exposed in the crystal structure, suggesting that a conformational change occurs in CPR that exposes the FMN-binding domain for binding with CYPs. ${ }^{19-21)}$ Simulation studies also suggested the occurrence of such a conformational change. ${ }^{19,22)}$

On the other hand, the role of the membrane anchor in the catalytic function of CPR remains undefined. The primary function of the anchor has been recognized to be mooring CPR to the membrane. CYP is also a membrane protein, and the anchor orients CPR for association with CYP. ${ }^{23-25)}$ This association protects FMN from exposure to water and suppresses the generation of reactive oxygen species by CPR. ${ }^{24}$ However, CPR that has been solubilized with detergents can still donate electrons to CYP. ${ }^{26,27)}$ When the membrane anchor was truncated by trypsin digestion, activity of CPR for cytochrome $c$ (cyt $c$ ) was not disturbed ${ }^{28,29)}$ while activity for CYP was diminished. ${ }^{30-32)}$ By contrast, CYP could be reduced electrochemically via the FMN domain, ${ }^{33)}$ suggesting that the membrane anchor is not required for electron transfer from CPR to CYP.

In addition to the electron transfer activity of CPR described above, the potential role of the membrane anchor in the metabolizing reaction of CYPs also remains unknown. For example, CYPs express mono-oxygenase activity with yeast CPR in which the membrane anchor was truncated, ${ }^{32)}$ while a chimeric CPR connected with the anchor domain of cytochrome $b_{5}$ could catalyze hydroxylation with CYP4A1 and 17A but not with CYP3A4. ${ }^{34)}$ In this work, we focus on the role of 
the membrane anchor of human CPR in the drug metabolism of CYP. We reported previously that human CYP2C19 is fully metabolically active even when its N-terminal membrane anchor is truncated. ${ }^{35,36)}$ Truncated CYP2C19 is soluble, but can associate with the membrane, thus allowing us to assess the role of the CPR anchor with this CYP in the presence and absence of phospholipids. We constructed a truncated human CPR in which the N-terminal 60 amino acids are removed $(\triangle 60-C P R)$, and investigated the effect of the truncation on the binding, electron transfer, and drug metabolizing properties of CYP2C19 to propose a novel function for the CPR membrane anchor.

\section{Experimental}

Expression and Purification of CPR The human $C P R$ gene harbored in the pET15b vector was kindly provided by Dr. Noritaka Ariyoshi, Chiba University Hospital. The $460-C P R$ gene was produced by PCR using the $C P R$ gene as a template and the following primers: $\triangle 60-\mathrm{CPR}-\mathrm{F}$, 5'-ACCCATATGCAG ACA TTG ACC TCC TCT GTC AG-3'; $\triangle 60-\mathrm{CPR}-\mathrm{R}, \quad 5^{\prime}$-AAAGGATCCCTA GCT CCA CAC GTC CAG G-3' (restriction sites are underlined). The wild-type (WT) and $\triangle 60-C P R$ genes were digested with NdeI and BamHI (New England Biolabs) and ligated into a pCold I vector (TaKaRa, Japan). The constructed expression vectors were transformed into BL21 Gold (DE3) Escherichia coli competent cells (Stratagene). The $E$. coli cells were cultured at $37^{\circ} \mathrm{C}$ in $\mathrm{LB}$ medium containing ampicillin $(100 \mu \mathrm{g} / \mathrm{mL})$. Protein expression was induced by temperature downshift from 37 to $15^{\circ} \mathrm{C}$ when the turbidity at $600 \mathrm{~nm}$ reached 0.7 , after which cell culture was continued for an additional $24 \mathrm{~h}$. The cells were collected by centrifugation $(3000 \times \boldsymbol{g})$ and frozen at $-80^{\circ} \mathrm{C}$ overnight. The frozen cells were thawed and resuspended in a buffer $(10 \mathrm{~mL} / \mathrm{g}$ wet cell) containing $100 \mathrm{~mm}$ potassium phosphate $(\mathrm{pH} 7.4)$, $0.1 \mathrm{~mm}$ ethylenediaminetetraacetic acid (EDTA), 20\% glycerol, $100 \mathrm{~mm} \mathrm{NaCl}$, and $30 \mu \mathrm{g} / \mathrm{mL}$ lysozyme. The resuspended solution was stirred at $4^{\circ} \mathrm{C}$ overnight. The suspension was subjected to a French Press (EmulsiFlex-C5, Avestin), and Triton $\mathrm{X}-100$ was added to a final concentration of $1 \%(\mathrm{v} / \mathrm{v})$. The solution was stirred for $2 \mathrm{~h}$, centrifuged at $30000 \times \boldsymbol{g}$ for $1 \mathrm{~h}$ at $4^{\circ} \mathrm{C}$, and the supernatant was purified with a $2^{\prime} 5^{\prime}$-ADP Sepharose 4B column (GE Healthcare, U.S.A.), as reported previously. ${ }^{37)}$ The CPR protein was eluted by 2 '-AMP step gradients and confirmed by sodium dodecyl sulfate-polyacrylamide gel electrophoresis (SDS-PAGE). The CPR fractions were concentrated with an Amicon Ultra-15 centrifugal filter unit $(50 \mathrm{kDa}$ MWCO, Merck, Millipore, U.S.A.) and washed with $100 \mathrm{~mm}$ KPi buffer (100 mm potassium phosphate, $0.1 \mathrm{~mm}$ EDTA, and $20 \%$ glycerol, $\mathrm{pH} 7.4$ ) to remove 2 -AMP completely. Finally, the concentration of CPR was estimated spectrophotometrically (DU-600, Beckman-Coulter, U.S.A.) by the addition of a trace amount of potassium ferricyanide to oxidize CPR fully, and the concentrations of WT- and $\triangle 60$-CPRs were determined with an extinction coefficient $\left(21.4 \mathrm{~mm}^{-1} \mathrm{~cm}^{-1}\right) .{ }^{29,38)}$ To evaluate the drug metabolism activities of CPR, we used CYP2C19, which was expressed and purified as reported previously. ${ }^{35,36)}$

Reduction Activity of CPR for Cyt $c$ The electron transfer activity of CPR was evaluated by reduction of cyt $c$ at $37^{\circ} \mathrm{C}$ in $100 \mathrm{~mm} \mathrm{KPi}$ buffer following procedures reported previously $^{39)}$ with slight modifications. Briefly, a solution containing $50 \mu \mathrm{M}$ ferric cyt $c$ (Sigma, horse heart) and $0.1 \mathrm{~nm}$ $\mathrm{CPR}$ (WT or $\Delta 60$ ) was incubated for $3 \mathrm{~min}$ at $37^{\circ} \mathrm{C}$, and NADPH solution in its regenerating system $(350 \mu \mathrm{M} N A D P H$, $880 \mu \mathrm{M}$ glucose-6-phosphate, $880 \mu \mathrm{M} \mathrm{MgCl}_{2}$, and 2 units $/ \mathrm{mL}$ glucose-6-phosphate dehydrogenase) was added to start the reaction. The rate of cyt $c$ reduction was estimated by absorbance change at $550 \mathrm{~nm}$, which is characteristic of ferrous cyt c. Binding affinity of CPR for NADPH was also evaluated using various amounts of NADPH $(1,3,5,10$, and $20 \mu \mathrm{M})$ in the regenerating system according to a previously reported method. ${ }^{16,40)}$

Drug Metabolizing Activity of CYP2C19 With the purified WT- and $\triangle 60-C P R$, we evaluated the metabolizing activities of CYP2C19 for four drugs, amitriptyline (AMT), imipramine (IMP), omeprazole (OPZ), and lansoprazole (LPZ). We previously reported several kinetic parameters for the metabolism of AMT and IMP with purchased CPR (SigmaAldrich). ${ }^{35,36)}$ The drug metabolizing activities of WT- and $\triangle 60$-CPRs were estimated in a similar manner using UPLC (Acquity H-class, Waters) ${ }^{36)}$ in the absence or presence of $30 \mu \mathrm{g} / \mathrm{mL}$ 1,2-dilauroyl-sn-glycero-3-phosphocholine (DLPC).

Binding Affinity of CPR for CYP2C19 The binding affinity of CPR for CYP2C19 was evaluated by surface plasmon resonance technique (Biacore T200, GE Healthcare). CPR was immobilized onto an NTA sensor chip to give $c a$. 200 resonance units (RU). CYP2C19 was prepared in a running buffer containing $10 \mathrm{~mm}$ 2-[4-(2-hydroxyethyl)piperazin-1-yl]ethanesulfonic acid (HEPES) ( $\mathrm{pH} 7.4), 150 \mathrm{~mm} \mathrm{NaCl}, 50 \mu \mathrm{M}$ EDTA, and $0.05 \%$ Surfactant P20. Subsequently, the CYP solution was injected at a flow rate of $30 \mu \mathrm{L} / \mathrm{min}$ for $2 \mathrm{~min}$, and dissociation was monitored for $3 \mathrm{~min}$ at $25^{\circ} \mathrm{C}$. The effect of drugs on CPR-CYP binding was also investigated with running buffer containing each drug at a concentration $c a$. 10 times higher than the $K_{\mathrm{m}}$ value (more than $90 \%$ of the CYP was saturated). Finally, the sensorgram thus obtained was analyzed with a two-state binding model using Biacore T200 software version 1.0 (GE Healthcare). This model consists of the CPRCYP binding step (with association $\left(k_{\mathrm{a} 1}\right)$ and dissociation $\left(k_{\mathrm{d} 1}\right)$ rate constants), followed by a conformational change of the complex (with two rate constants $k_{\mathrm{a} 2}$ and $k_{\mathrm{d} 2}$ ). The total equilibrium dissociation constant $K_{\mathrm{D}}$ was calculated using Eq. 1:

$$
K_{\mathrm{D}}=k_{\mathrm{d} 1} k_{\mathrm{d} 2} / k_{\mathrm{a} 1}\left(k_{\mathrm{d} 2}+k_{\mathrm{a} 2}\right)
$$

Reduction Rate of CYP2C19 under a CO Atmosphere To measure electron transfer activity of CPR to CYP2C19, $1 \mu \mathrm{M} \mathrm{CPR}, 1 \mu \mathrm{M} \mathrm{CYP} 2 \mathrm{C} 19$, and $30 \mu \mathrm{g} / \mathrm{mL}$ DLPC (if necessary) were incubated in $100 \mathrm{~mm} \mathrm{KPi}$ buffer for $5 \mathrm{~min}$ at $37^{\circ} \mathrm{C}$ in a cuvette. The cuvette was sealed with a rubber septum and saturated with 1 atm of $\mathrm{CO}$ gas. The electron transfer reaction was started by introducing NADPH regenerating solution (100 $\mu \mathrm{M}$ NADPH, $250 \mu \mathrm{M}$ glucose-6-phosphate, $250 \mu \mathrm{M} \mathrm{MgCl}_{2}$, and 2 units $/ \mathrm{mL}$ glucose-6-phosphate dehydrogenase) in which the NADPH concentration remained constant. The reduction rate of CYP2C19 was measured similarly in the presence of each drug at a concentration $c a$. 10 times higher than the $K_{\mathrm{m}}$ value. The reduction rates of CYP2C19 were determined by the initial absorbance change at $450 \mathrm{~nm}$ originating from the $\mathrm{CO}$ adduct of ferrous CYP.

Estimation of Uncoupling Reaction To estimate the uncoupling reaction and evaluate efficiency of electron con- 

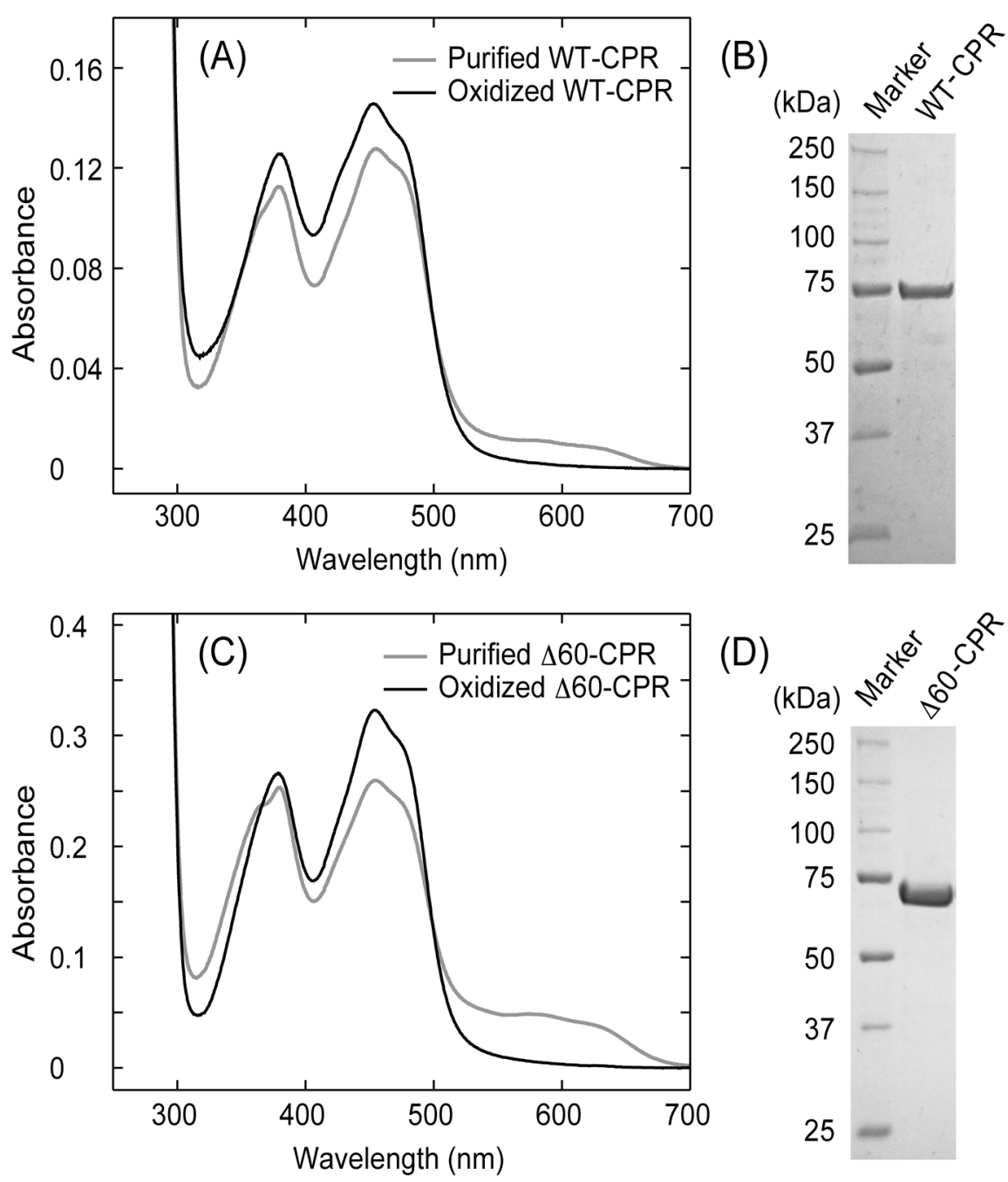

Fig. 1. UV-Visible Absorption Spectra and SDS-PAGE of Purified WT-CPR (A, B) and $\triangle 60-C P R(C, D)$

The spectra of purified (gray lines) and ferricyanide-oxidized CPRs (black lines) are shown. Purified CPRs were electrophoresed and stained by Coomassie brilliant blue.

sumption, we quantitated the amounts of produced $\mathrm{H}_{2} \mathrm{O}_{2}$ and drug metabolites. Sample solution was prepared as described in the previous section but under aerobic conditions. The drug metabolizing reaction was initiated by the addition of $100 \mu \mathrm{M} \mathrm{NADPH}$ at $37^{\circ} \mathrm{C}$, and NADPH consumption was determined by following its absorbance at $340 \mathrm{~nm}$. After complete consumption, the amount of metabolites was determined by UPLC. $\mathrm{H}_{2} \mathrm{O}_{2}$ was generated as a by-product by the metabolizing reaction and was detected fluorometrically with a horseradish peroxidase-resorufin system (Enzo). Although the reduction equivalent of NADPH (2 eq) is consumed to produce a metabolite (2 eq), uncoupling of the metabolism will generate either $\mathrm{H}_{2} \mathrm{O}_{2}$ (2 eq) or water (1 eq) via the superoxide/ peroxide shunt and oxidase shunt pathways, respectively (Fig. 5). Therefore, the amount of water produced by the oxidase shunt can be estimated by Eq. $2^{41-43)}$ :

$$
\left[\mathrm{H}_{2} \mathrm{O}\right]=\left([\mathrm{NADPH}]-[\text { metabolites }]-\left[\mathrm{H}_{2} \mathrm{O}\right]\right) / 2
$$

\section{Results}

Purification of CPRs and Reductase Activity for Cyt $c$ WT-CPR and $\triangle 60-C P R$ were expressed and highly purified as confirmed by SDS-PAGE (Figs. 1B, D). The absorption spectra of WT-CPR are shown in Fig. 1A. CPR contains two flavins (FAD and FMN), which exhibit characteristic peaks at $380 \mathrm{~nm}$ and $455 \mathrm{~nm}$. A weak and broad band of purified CPR near $600 \mathrm{~nm}$ is assignable to a half-reduced semiquinone form of flavins, ${ }^{29,37,38)}$ which disappeared completely following the addition of a trace amount of ferricyanide (oxidized spectrum). The spectrum of $\triangle 60-C P R$ (Fig. 1C) was similar to that of WT-CPR, indicating that both WT- and $\triangle 60-C P R$ have similar structural features around the two flavins.

We subsequently evaluated the electron transfer activity of both CPRs for cyt $c$. The production of ferrous cyt $c$ was traced, and the reduction rate was obtained from the slope (Fig. 2, inset). The rates were estimated with various NADPH concentrations (Fig. 2) and analyzed with the Michaelis-Menten equation ${ }^{40)}$ to give the maximal activity and $K_{\mathrm{m}}{ }^{\mathrm{NADPH}}$. WT- and $\triangle 60-\mathrm{CPR}$ were evaluated similarly, and the maximal activity of WT-CPR $(2.43 \pm 0.02 \mu \mathrm{mol} / \mathrm{min} / \mathrm{nmol}$ of CPR) was almost the same as that reported previously. ${ }^{44)}$ Interestingly, the activity of $\Delta 60-\mathrm{CPR}(2.63 \pm 0.11 \mu \mathrm{mol} / \mathrm{min} /$ nmol of CPR) was almost equal to that of WT-CPR, indicating that $\triangle 60-\mathrm{CPR}$ can also catalyze electron transfer (i.e., $\mathrm{NADPH} \rightarrow \mathrm{FAD} \rightarrow \mathrm{FMN} \rightarrow$ cyt $c$ ). Moreover, WT- and $\Delta 60-\mathrm{CPR}$ 


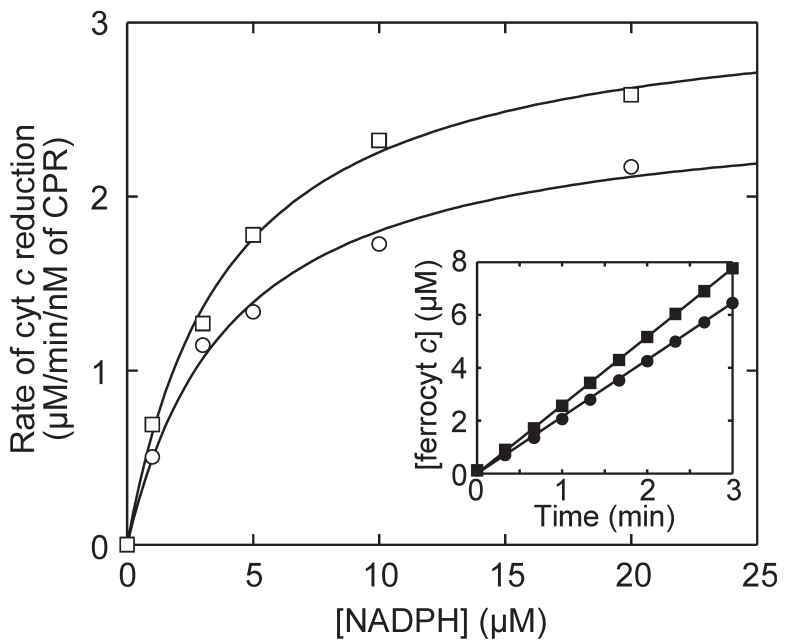

Fig. 2. Reduction Kinetics of CPRs for Ferricytochrome $c$

The inset shows the time course of ferrocyt $c$ production by WT-CPR (filled circles) and $\triangle 60-\mathrm{CPR}$ (filled squares) with $20 \mu \mathrm{M}$ NADPH. The reduction rates were calculated from the slopes and plotted against NADPH concentrations. A solution containing ferric cyt $c(100 \mu \mathrm{M})$ and either WT-CPR (open circles) or $\Delta 60-\mathrm{CPR}$ (open squares) $(1 \mathrm{nM})$ was pre-incubated for $3 \mathrm{~min}$ at $37^{\circ} \mathrm{C}$ in a buffer containing $100 \mathrm{~mm}$ potassium phosphate, $0.1 \mathrm{~mm}$ EDTA, and $20 \%$ glycerol $(\mathrm{pH} \mathrm{7.4)}$, and the reduction was initiated by the addition of NADPH.

showed similar $K_{\mathrm{m}}{ }^{\mathrm{NADPH}}$ values $(4.17$ and $3.92 \mu \mathrm{M})$, and it is now clear that both CPR preparations have nearly the same electron transfer activity. Therefore, truncation of the CPR membrane anchor minimally affects reduction of cyt $c$. In fact, Das and Sligar reported previously that truncation of the membrane anchor had no effect on the redox potential of FMN ${ }^{45)}$ consistent with our results with $\triangle 60-\mathrm{CPR}$.

Effect of the Membrane Anchor on Drug Metabolism Because $\triangle 60-\mathrm{CPR}$ was found to be active similarly to WTCPR with respect to cyt $c$ reduction, we measured drug metabolism of CYP2C19 with both CPRs. It has been reported that deletion of the CPR membrane anchor impairs the drug metabolism activity of several CYP isoforms. ${ }^{30-32,34)}$ Four drugs (AMT, IMP, OPZ, and LPZ) were employed, and the reaction velocities were plotted against the drug concentrations (Fig. 3). For each drug, WT-CPR showed the highest velocity in the presence of DLPC (filled circles), as expected. The kinetic parameters $\left(K_{\mathrm{m}}\right.$ and $\left.V_{\max }\right)$ were evaluated with the Michaelis-Menten equation, and the intrinsic clearance $\left(C L_{\text {int }}\right)$, an index of metabolic efficacy, was calculated $\left(C L_{\text {int }}=V_{\max } / K_{\mathrm{m}}\right)$. The values obtained are summarized in Table 1. OPZ and LPZ were good substrates for CYP2C19/WT-CPR. In the presence of DLPC, both the $V_{\max }$ and $K_{\mathrm{m}}$ values for LPZ were approximately one-order of magnitude lower than those for OPZ, giving nearly equal $C L_{\text {int }}$ values $(248$ and $369 \mathrm{~mL} /$ $\mathrm{min} / \mu \mathrm{mol})$. While the metabolizing activities of WT-CPR were impaired in the absence of DLPC $(119$ and $88 \mathrm{~mL} / \mathrm{min} /$ $\mu \mathrm{mol})$, they were completely lost with $\Delta 60-\mathrm{CPR}$, irrespective of DLPC content. This result is consistent with previous reports $^{30-32,34)}$ that stressed the importance of the CPR membrane anchor in drug metabolism.

AMT and IMP were also good substrates for CYP2C19/ WT-CPR. Although the $V_{\max }$ and $K_{\mathrm{m}}$ values were relatively larger than those for $\mathrm{OPZ}$ and $\mathrm{LPZ}$, the $C L_{\text {int }}$ values were almost the same $(376 \mathrm{~mL} / \mathrm{min} / \mu \mathrm{mol}$ for $\mathrm{AMT})$ or much larger $(1300 \mathrm{~mL} / \mathrm{min} / \mu \mathrm{mol}$ for IMP). As described above for OPZ and LPZ, the metabolizing activities with WT-CPR were im- paired in the absence of DLPC (178 and $326 \mathrm{~mL} / \mathrm{min} / \mu \mathrm{mol})$. In contrast to the cases for OPZ and LPZ, however, the metabolizing activities with $\triangle 60-\mathrm{CPR}$ were not lost, even in the absence of DLPC (Table 1). These results were surprising and in contrast with those observed for OPZ and LPZ. These findings indicate unequivocally that the role of membrane anchor is variable with the type of drug, and suggested that we would be able to reveal the role of the membrane anchor by exploring the properties of metabolism of the AMT/IMP and OPZ/ LPZ pairs.

CPR Binding to CYP2C19 Because drug metabolism by CYPs requires electrons that are transferred from CPR to CYPs, the membrane anchor might have a role in the CYPCPR binding that is affected in a drug-specific manner. Therefore, the binding affinity of CPR and CYP2C19 was examined with surface plasmon resonance technique in the presence or absence of different drugs. WT- and $\triangle 60-\mathrm{CPR}$ were immobilized on a sensor chip NTA with an N-terminal $\mathrm{His}_{6}$ tag, and binding of CYP2C19 was evaluated with a dissociation constant $\left(K_{\mathrm{D}}\right)$. Figure 4 shows the association and dissociation kinetics of CYP2C19 with WT-CPR (A) and $\triangle 60-\mathrm{CPR}$ (B). The sensorgram was analyzed with a two-state binding model, and the total dissociation constants were calculated (Table 2). Interestingly, in the absence of drugs, the WT- and $\triangle 60-\mathrm{CPRs}$ showed similar $K_{\mathrm{D}}$ values $(1.99,1.54 \mu \mathrm{M})$, implying that $\triangle 60-\mathrm{CPR}$ is fully active in binding with CYP2C19.

In the presence of a large amount of drugs, the $K_{\mathrm{D}}$ value for WT-CPR was reduced, indicating that the binding of each drug to CYP2C19 raised its affinity for WT-CPR. This strongly suggests that drug binding to CYP2C19 modifies its structure to facilitate binding to WT-CPR. On the other hand, the drugs had distinct effects on binding to $\triangle 60-\mathrm{CPR}$. The $K_{\mathrm{D}}$ value of $1.54 \mu \mathrm{M}$ was reduced in the presence of AMT and IMP, while it was increased by OPZ and LPZ. As a result, CYP2C19 preferred binding to $\triangle 60-C P R$ in the presence of AMT and IMP, but to WT-CPR in the presence of OPZ and LPZ. Although the binding preference of CPRs was dependent on the drug type, the effect of drugs was not very large and could not explain the loss of $\triangle 60$-CPR-mediated metabolism of OPZ and LPZ.

Electron Transfer under a CO Atmosphere Because both WT- and $\triangle 60-\mathrm{CPRs}$ could bind to CYP2C19, the loss of $\triangle 60$-CPR-mediated metabolism of OPZ and LPZ (Table 1) might be attributed to subsequent electron transfer steps. If electrons were successfully transferred from CPRs to CYP2C19, the ferrous heme thus produced would be trapped as a ferrous- $\mathrm{CO}$ adduct under a $\mathrm{CO}$ atmosphere. As shown in Fig. 5A, the formation of ferrous-CO was traced via its characteristic absorption peak at $450 \mathrm{~nm}$, and the electron transfer rate was estimated from the initial slope.

The electron transfer rates are summarized in Fig. 5B. In the absence of drugs, WT-CPR and $\triangle 60-\mathrm{CPR}$ showed similar electron transfer rates irrespective of the DLPC content, indicating that both WT-CPR and $\triangle 60-C P R$ are competent in electron transfer to CYP2C19. In the presence of any of the drugs, WT-CPR showed the highest electron transfer rate with DLPC. Except for AMT, the activity of WT-CPR was independent of DLPC concentration. In the case of $\triangle 60-C P R$, the rate was relatively low with OPZ, but the difference in electron transfer rate was minimal for LPZ. Therefore, the loss of drug metabolizing activity of $\triangle 60-\mathrm{CPR}$ does not result from 

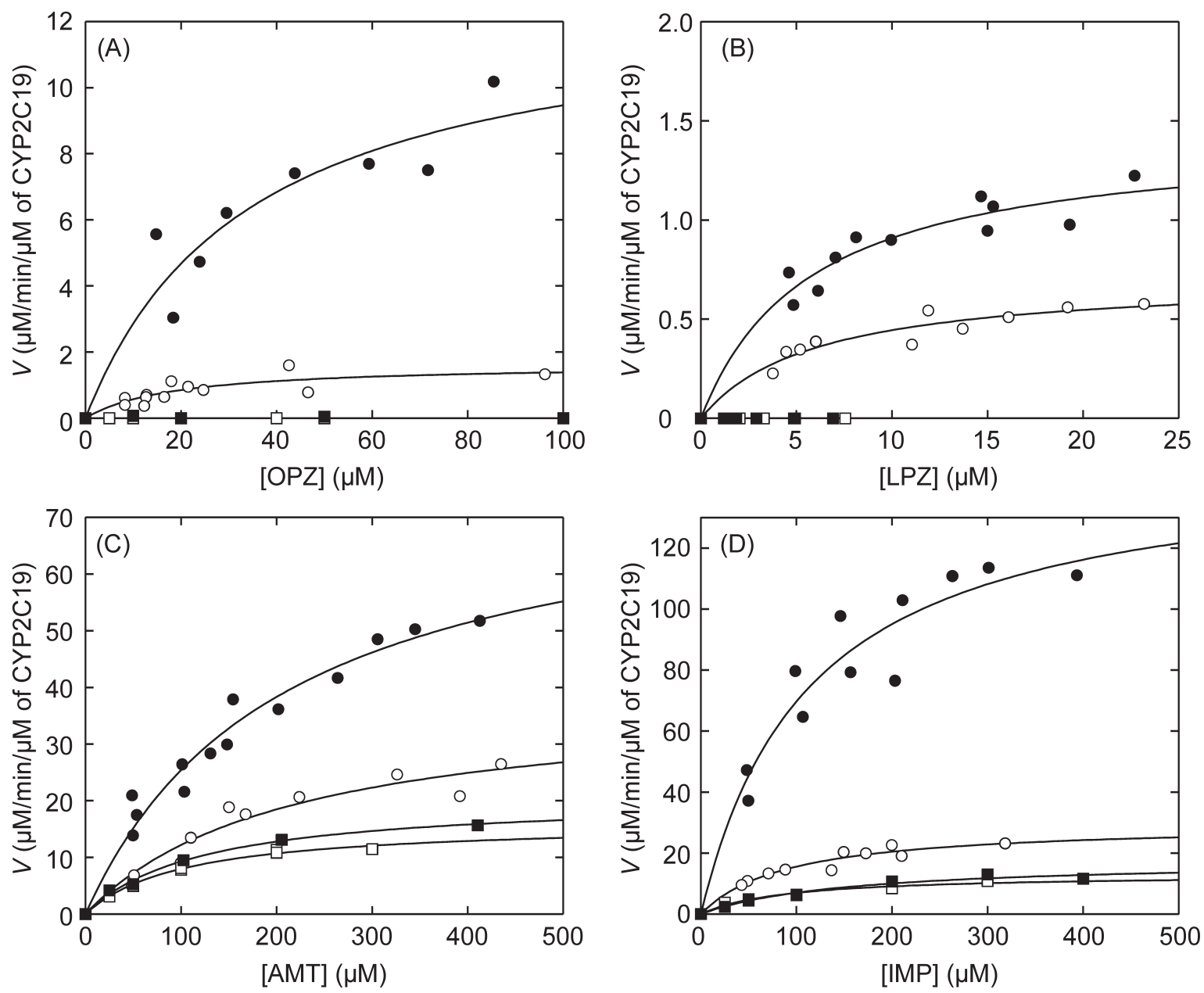

Fig. 3. Drug Metabolizing Activity of CYP2C19 with WT- and $\triangle 60-C P R$

Activities were measured in the presence of various concentrations of OPZ (A), LPZ (B), AMT (C), and IMP (D). The reaction velocities with WT-CPR (circles) or $\triangle 60$-CPR (squares) were plotted against various concentrations of each drug in the presence (filled symbols) or absence (open symbols) of $30 \mu \mathrm{g} / \mathrm{mL} \mathrm{DLPC}$.

Table 1. Metabolizing Activities of CYP2C19 for AMT, IMP, OPZ, and LPZ

\begin{tabular}{|c|c|c|c|c|c|}
\hline Drugs & CPRs & DLPC & $V_{\max }(\mu \mathrm{M} / \min / \mu \mathrm{M}$ of CYP $)$ & $K_{\mathrm{m}}(\mu \mathrm{M})$ & $C L_{\text {int }}(\mathrm{mL} / \mathrm{min} / \mu \mathrm{mol}$ of CYP $)$ \\
\hline \multirow[t]{4}{*}{ AMT } & WT & + & $78 \pm 7$ & $208 \pm 39$ & $376 \pm 38$ \\
\hline & & - & $38 \pm 6$ & $214 \pm 64$ & $178 \pm 30$ \\
\hline & $\Delta 60$ & + & $21 \pm 1$ & $122 \pm 11$ & $169 \pm 11$ \\
\hline & & - & $16 \pm 1$ & $104 \pm 11$ & $157 \pm 10$ \\
\hline \multirow[t]{4}{*}{ IMP } & WT & + & $149 \pm 17$ & $114 \pm 33$ & $1300 \pm 240$ \\
\hline & & - & $30 \pm 3$ & $91 \pm 24$ & $326 \pm 55$ \\
\hline & $\Delta 60$ & + & $18 \pm 2$ & $152 \pm 47$ & $116 \pm 22$ \\
\hline & & - & $13 \pm 2$ & $89 \pm 26$ & $148 \pm 29$ \\
\hline \multirow[t]{4}{*}{$\mathrm{OPZ}$} & WT & + & $13 \pm 2$ & $35 \pm 15$ & $369 \pm 98$ \\
\hline & & - & $1.6 \pm 0.3$ & $19 \pm 9$ & $88 \pm 25$ \\
\hline & $\Delta 60$ & + & $c a .0$ & N.D. & N.D. \\
\hline & & - & ca. 0 & N.D. & N.D. \\
\hline \multirow[t]{4}{*}{ LPZ } & WT & + & $1.4 \pm 0.1$ & $5.8 \pm 1.5$ & $248 \pm 43$ \\
\hline & & - & $0.71 \pm 0.07$ & $6.0 \pm 1.7$ & $119 \pm 22$ \\
\hline & $\Delta 60$ & + & $c a .0$ & N.D. & N.D. \\
\hline & & - & ca. 0 & N.D. & N.D. \\
\hline
\end{tabular}

The activities were measured with WT- and $\triangle 60$-CPR in the presence and absence of DLPC. N.D., not determined.

altered electron transfer activity.

Shunt Pathway in Drug Metabolism In Fig. 6, the generally accepted reaction cycle of $\mathrm{CYP}^{39,46)}$ is depicted. CPR has been suggested to supply electrons to substrate-bound and superoxide-bound ferric CYPs. These electrons are utilized effectively to mono-oxygenate a substrate $(\mathrm{RH})$ to complete the cycle. Although drug metabolism is mediated primarily via this main pathway, recent studies have focused on 

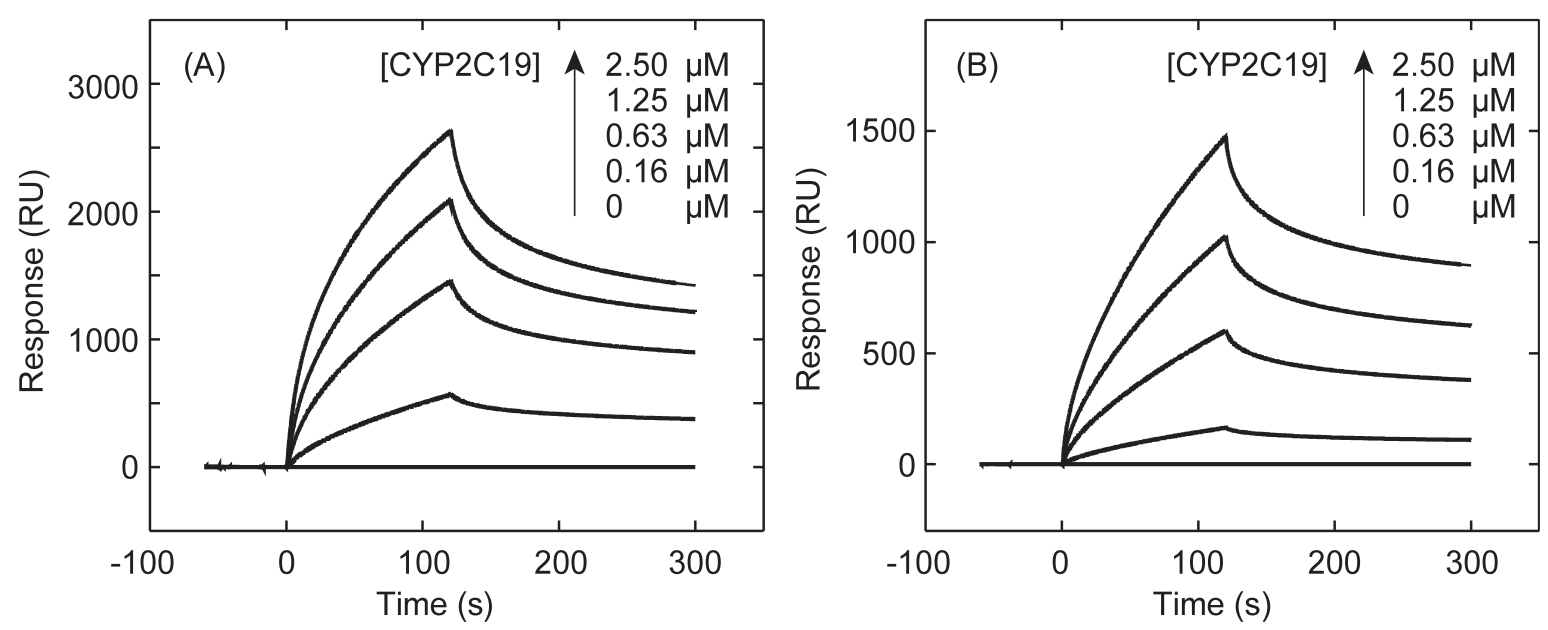

Fig. 4. Sensorgrams of CYP2C19 Binding to WT-CPR (A) and $\triangle 60-C P R(B)$ in the Presence of LPZ

Analyte solutions contained LPZ $(100 \mu \mathrm{m})$ and CYP2C19 $(0.16,0.63,1.25$, or $2.50 \mu \mathrm{m})$ and were flowed over a sensor chip on which the CPRs were immobilized. The flow rate was $30 \mu \mathrm{L} / \mathrm{min}$, and contact and dissociation were monitored for 120 and $180 \mathrm{~s}$, respectively. The sensorgrams were analyzed with Biacore T200 Evaluation Software version 1.0 assuming a two-state reaction.

Table 2. Binding Rates and Affinity of CPRs for CYP2C19 in the Absence and Presence of AMT (1000 $\mu \mathrm{M})$, IMT (1000 $\mu \mathrm{M})$, OPZ (400 $\mu \mathrm{M})$ and LPZ $(100 \mu \mathrm{M})$

\begin{tabular}{|c|c|c|c|c|c|c|}
\hline Drugs & CPRs & $k_{\mathrm{a} 1}\left(\mathrm{mM}^{-1} \mathrm{~s}^{-1}\right)$ & $k_{\mathrm{d} 1}\left(10^{-3} \mathrm{~s}^{-1}\right)$ & $k_{\mathrm{a} 2}\left(10^{-3} \mathrm{~s}^{-1}\right)$ & $k_{\mathrm{d} 2}\left(10^{-3} \mathrm{~s}^{-1}\right)$ & $K_{\mathrm{D}}(\mu \mathrm{M})$ \\
\hline \multirow[t]{2}{*}{ None } & WT & $1.97 \pm 0.19$ & $35.2 \pm 2.8$ & $16.4 \pm 1.0$ & $2.07 \pm 0.03$ & $1.99 \pm 0.09$ \\
\hline & $\Delta 60$ & $1.86 \pm 0.16$ & $40.2 \pm 4.1$ & $23.1 \pm 0.8$ & $1.67 \pm 0.04$ & $1.54 \pm 0.20$ \\
\hline \multirow[t]{2}{*}{ AMT } & WT & $1.96 \pm 0.24$ & $33.1 \pm 0.8$ & $21.8 \pm 0.1$ & $1.32 \pm 0.08$ & $1.31 \pm 0.34$ \\
\hline & $\Delta 60$ & $2.38 \pm 0.26$ & $26.5 \pm 0.2$ & $23.7 \pm 0.4$ & $1.26 \pm 0.07$ & $0.48 \pm 0.02$ \\
\hline \multirow[t]{2}{*}{ IMP } & WT & $3.39 \pm 0.23$ & $36.9 \pm 3.2$ & $26.2 \pm 0.8$ & $1.29 \pm 0.05$ & $0.49 \pm 0.05$ \\
\hline & $\Delta 60$ & $2.78 \pm 0.30$ & $16.9 \pm 7.6$ & $16.4 \pm 4.0$ & $0.62 \pm 0.01$ & $0.24 \pm 0.16$ \\
\hline \multirow[t]{2}{*}{$\mathrm{OPZ}$} & WT & $3.56 \pm 0.23$ & $43.9 \pm 13.2$ & $16.4 \pm 2.1$ & $1.30 \pm 0.01$ & $0.89 \pm 0.24$ \\
\hline & $\Delta 60$ & $1.72 \pm 0.63$ & $47.9 \pm 5.7$ & $17.7 \pm 1.2$ & $1.62 \pm 0.01$ & $2.35 \pm 0.29$ \\
\hline \multirow[t]{2}{*}{ LPZ } & WT & $2.93 \pm 0.44$ & $16.5 \pm 0.9$ & $16.5 \pm 0.9$ & $2.12 \pm 0.01$ & $0.64 \pm 0.04$ \\
\hline & $\Delta 60$ & $1.76 \pm 0.60$ & $40.5 \pm 3.6$ & $18.1 \pm 0.5$ & $1.77 \pm 0.04$ & $2.09 \pm 0.16$ \\
\hline
\end{tabular}

uncoupling pathways that do not produce any metabolites except waste electrons. These uncoupling pathways are (1) an auto-oxidation shunt to produce superoxide, (2) a peroxide shunt to produce $\mathrm{H}_{2} \mathrm{O}_{2}$, and (3) an oxidase shunt to produce water. ${ }^{47-49)}$ The superoxide in (1) is short-lived and readily converted to $\mathrm{H}_{2} \mathrm{O}_{2}$ under physiological conditions. Therefore, we measured by-product $\mathrm{H}_{2} \mathrm{O}_{2}$ produced via pathway 1 and 2 and compared the amounts produced by WT- and $\triangle 60-\mathrm{CPR}$ in the presence and absence of drugs and DLPC. The produced $\mathrm{H}_{2} \mathrm{O}_{2}$ and metabolites were quantitated after $100 \mu \mathrm{M}$ NADPH was completely consumed by drug metabolism, as described in Experimental.

Both WT- and $\triangle 60$-CPR produced considerable amounts of $\mathrm{H}_{2} \mathrm{O}_{2}$, even in the absence of drug (Table 3), indicating that the electrons of NADPH are wasted by CPR/CYP at the basal level. In the presence of each drug, WT-CPR produced the drug metabolite along with a lower amount of $\mathrm{H}_{2} \mathrm{O}_{2}$, although the amount of $\mathrm{H}_{2} \mathrm{O}_{2}$ produced was dependent on the drug type. Following the addition of DLPC, the amount of $\mathrm{H}_{2} \mathrm{O}_{2}$ was further diminished, with an increase in the production of metabolites, indicating that the electrons were efficiently utilized in drug metabolism. More specifically, the production of $\mathrm{H}_{2} \mathrm{O}_{2}$ by the superoxide/peroxide shunts greatly impairs drug metabolism.

In the case of $\triangle 60-\mathrm{CPR}$, the amount of $\mathrm{H}_{2} \mathrm{O}_{2}$ produced increased relative to WT-CPR with a concomitant decrease in metabolite production. The effect of DLPC was small, indicating that drug metabolism with $\triangle 60-\mathrm{CPR}$ is independent of the lipid membrane. AMT and IMP produced approximately half the amount of $\mathrm{H}_{2} \mathrm{O}_{2}(c a .20 \mu \mathrm{M})$ relative to basal levels (ca. $40 \mu \mathrm{M}$ ), with concomitant production of their metabolites. By contrast, metabolites of OPZ and LPZ were not detected with $\triangle 60-\mathrm{CPR}$, and this is consistent with the data shown in Table 1. It should be noted here that the amount of $\mathrm{H}_{2} \mathrm{O}_{2}$ produced was close to that of the basal level; in the absence of drug, approximately $42 \mu \mathrm{M}$ was produced by $\Delta 60-\mathrm{CPR}$ and remained almost unchanged in the presence of drug ( $\mathrm{ca} .40 \mu \mathrm{M}$ with OPZ and $c a .45 \mu \mathrm{M}$ with LPZ). Because the amount of water, and hence the contribution of the oxidase shunt, was calculated to be almost unaffected (approximately $30 \mu \mathrm{M}$ in all cases), the loss of $\triangle 60-C P R$-mediated metabolism of OPZ and LPZ can be attributed to shunt pathway 1 and/or 2 .

\section{Discussion}

Reductase Activity of $\triangle 60-C P R$ and Drug Metabolism To reveal the role of the CPR membrane anchor, we compared the activity of $\triangle 60-C P R$ with that of WT-CPR. Figure 1 shows that $\triangle 60-\mathrm{CPR}$ was highly purified and that its absorption spectrum was identical to that of WT-CPR in the oxidized state. In addition, $\triangle 60-\mathrm{CPR}$ could mediate electron transfer 

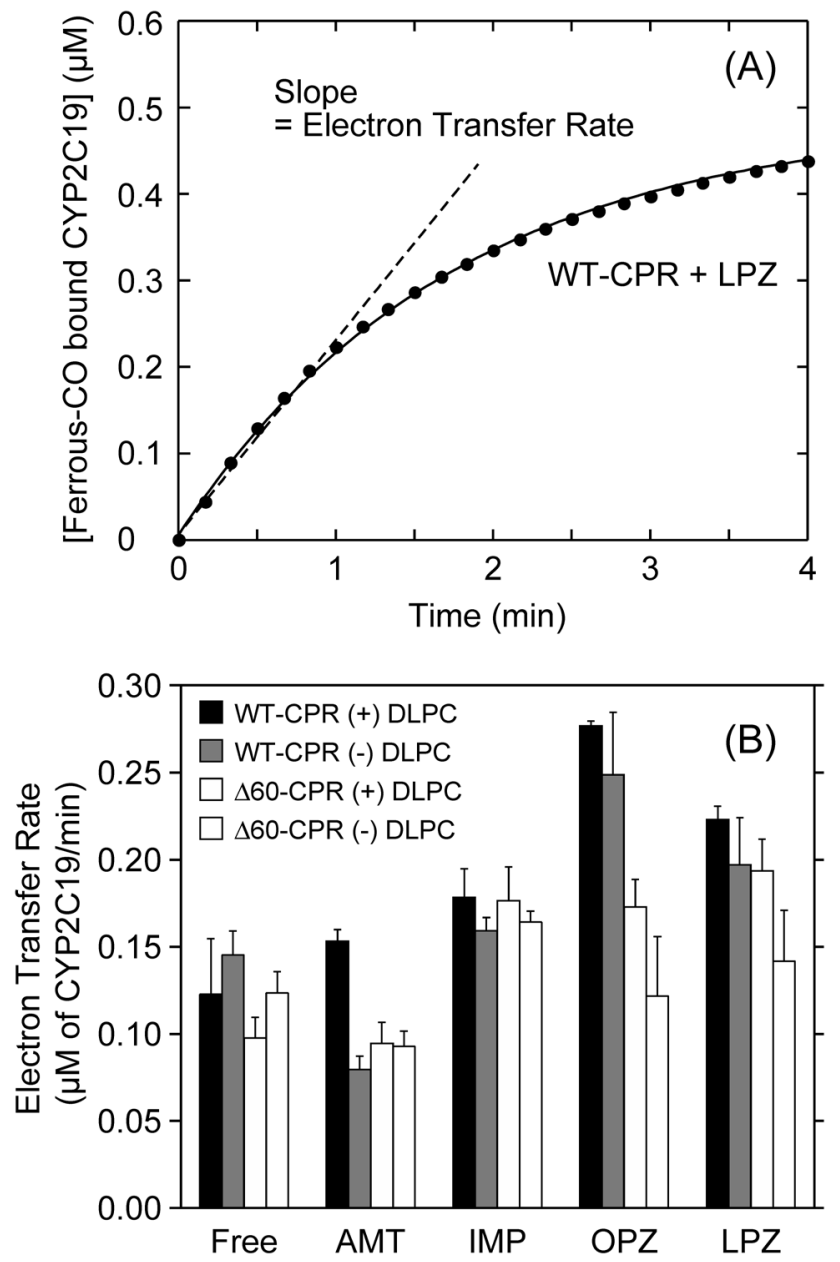

Fig. 5. Reduction of CYP2C19 by CPRs under a CO Atmosphere

Absorbance changes at $450 \mathrm{~nm}$ of ferrous-CO bound CYP2C19 $(1 \mu \mathrm{M})$ were monitored in the presence of $0.1 \mu \mathrm{M}$ CPR and $100 \mu \mathrm{m}$ NADPH under 1 atm CO in buffer containing $100 \mathrm{~mm}$ potassium phosphate, $0.1 \mathrm{~mm}$ EDTA, and $20 \%$ glycerol (pH 7.4) The reduction rate was estimated with the initial slope of the formation of ferrousCO bound CYP2C19, as typically observed in the presence of $100 \mu \mathrm{M} \mathrm{LPZ} \mathrm{(A).} \mathrm{The}$ reduction rates with WT-CPR or $\triangle 60-\mathrm{CPR}$ were determined in the absence or presence of AMT $(1000 \mu \mathrm{M})$, IMP $(1000 \mu \mathrm{M})$, OPZ $(400 \mu \mathrm{M})$, and LPZ $(100 \mu \mathrm{M})$ with or without $30 \mu \mathrm{g} / \mathrm{mL}$ DLPC (B).

from NADPH to ferric cyt $c$, and its activity was comparable to that of WT-CPR. Therefore, we then measured its drug metabolizing activity in detail, and compared it with that of WT-CPR.

WT-CPR attained the highest level of metabolizing activity by CYP2C19 in the presence of DLPC (Table 1). The activity of WT-CPR decreased in the absence of DLPC, but was not lost entirely. This residual activity might be attributed to phospholipids carried over from E. coli, the host cell used to express the enzyme. The residual activity for AMT/IMP metabolism, however, was almost equal to that of $\triangle 60-C P R$, and the activity of $\triangle 60-C P R$ was not affected by the presence of DLPC. Because the membrane anchor is truncated in $\triangle 60-C P R$ and should thus lose its membrane binding capacity, it is reasonable that its activity was insensitive to DLPC content. In other words, because the metabolism of AMT/ IMP does not require lipid membrane binding, both WT- and $\triangle 60-C P R s$ could mediate electron transfer and complete metabolism. The relatively low activity of $\triangle 60-\mathrm{CPR}$ would be attributed to its lack of membrane localization. WT-CPR is membrane-anchored, facilitating association with CYP2C19, which also resides on the membrane. ${ }^{23)}$ In the absence of phospholipids, however, both WT- and $\triangle 60$-CPRs were released from the membrane, and this would reduce the opportunity of the CPRs to associate with CYP2C19, resulting in low metabolizing activity.

CPR-CYP2C19 Binding and Electron Transfer As summarized in Table 2, WT- and $\triangle 60-\mathrm{CPR}$ showed similar binding affinity for CYP2C19 $(1.99,1.54 \mu \mathrm{M})$, indicating that $\triangle 60-\mathrm{CPR}$ possesses the residues necessary for binding. However, binding affinity was modified by the presence of drugs in a drug type-dependent manner. Because the drugs are substrates of CYP2C19, the differences in $K_{\mathrm{D}}$ should be attributed to properties of CYP2C19. The amino acids located on the C-helix that face CPR are highly conserved, and the C-helix of the CYPs is reported to change its conformation following drug binding. ${ }^{50)}$ Therefore, it is speculated that changes in the $K_{\mathrm{D}}$ may be attributed to conformational changes at the drug binding site in CYP2C19, with different drugs inducing different conformations of the C-helix at the surface.

The conformational change might be reflected in the reduction kinetics of CYP2C19, because the association is prerequisite to the electron transfer. As shown in Fig. 5, the effect of DLPC was minimal in every case; hence, membrane lipids do not contribute to the electron transfer rate. This is consistent with the finding that both CPRs could reduce cyt $c$ equally. In the cases of OPZ and LPZ, the electron transfer rate was reduced, while in the cases of AMT and IMP, the rate was reduced (AMT) or unchanged (IMP) (Fig. 5B). Therefore, the conformational modification of CYP2C19, if any, may not necessarily reflect the electron transfer rate.

Effect of Membrane Anchor on the Shunt Pathway As discussed above, the association between CPR and CYP2C19 and electron transfer are somewhat modified by truncation of the membrane anchor and are dependent on drug type. However, this cannot explain the loss of $\triangle 60$-CPR-mediated metabolism of OPZ and LPZ. Therefore, we studied the shunt pathway (Fig. 6) and found that deletion of the membrane anchor results in the wasting of electrons transferred by CPRs. The production of $\mathrm{H}_{2} \mathrm{O}_{2}$ was inversely correlated with the amount of metabolites (Table 3), and we revealed that the membrane anchor is prerequisite for CYP-mediated drug metabolism and that this effect depends on the drug type.

$\mathrm{H}_{2} \mathrm{O}_{2}$ is generated via shunt pathway 1 and/or 2 (Fig. 6), and the superoxo or hydroperoxo adduct of the heme should be impaired to drive the reaction cycle in the metabolism of OPZ and LPZ. Because both CPRs could bind with CYP2C19 (Table 2) and reduce CYP2C19 (Fig. 5), the metabolizing defect may have stemmed from the proton utilization. If the first proton is unavailable, the superoxo adduct will be lost via shunt 1 , and if the second proton is not supplied, the peroxo adduct will be lost via shunt 2. In either case, the proton defect should inhibit the heterolytic cleavage of the $\mathrm{O}-\mathrm{O}$ bond, ${ }^{48,51)}$ and compound I will not be formed. CYP2C19 has a large space for substrate binding, and many Thr residues are positioned there. ${ }^{52)}$ Because a bound drug and water network should occur in the same pocket, a complicated hydrogen bonding network in CYP2C19 might be modified by drug binding, depending on the drug type.

On the other hand, proximal residues of the heme are also considered to play an important role in the formation of compound I. In particular, the conserved Cys ligand of a heme is 


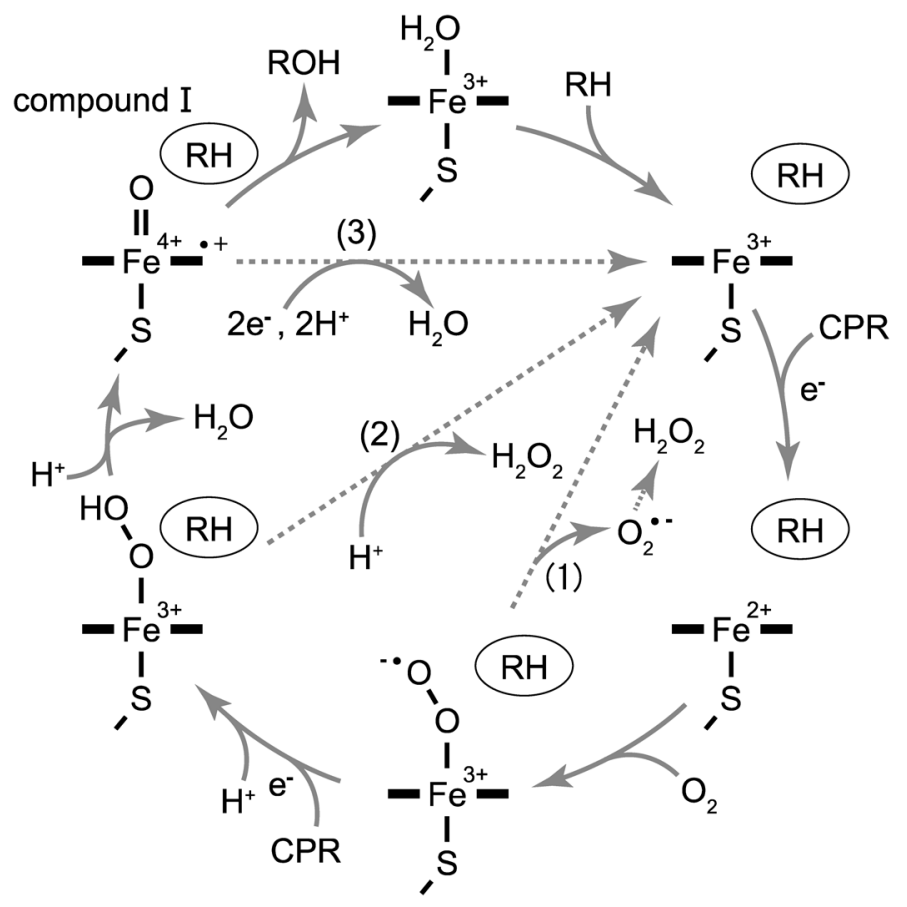

Fig. 6. Catalytic Cycle of CYP $\mathrm{P}^{46}$

One mole of a drug $(\mathrm{RH})$ is converted to its metabolite ( $\mathrm{ROH})$ with $1 \mathrm{~mol}$ of oxygen, 2 mol of electrons, and 2 mol of protons. The uncoupling reactions that waste electrons are classified into (1) auto-oxidation, (2) peroxide, and (3) oxidase shunts, which are depicted with dashed gray lines.

Table 3. Production of $\mathrm{H}_{2} \mathrm{O}_{2}, \mathrm{H}_{2} \mathrm{O}$, and Metabolites by Drug Metabolism

\begin{tabular}{|c|c|c|c|c|c|}
\hline Drugs & CPRs & DLPC & $\mathrm{H}_{2} \mathrm{O}_{2}(\mu \mathrm{M})$ & Metabolites $(\mu \mathrm{M})$ & $\mathrm{H}_{2} \mathrm{O}(\mu \mathrm{M})$ \\
\hline \multirow[t]{4}{*}{ None } & WT & + & $41.7 \pm 0.6$ & & 29.5 \\
\hline & & - & $39.2 \pm 0.5$ & & 30.0 \\
\hline & $\Delta 60$ & + & $42.5 \pm 2.6$ & & 31.1 \\
\hline & & - & $41.1 \pm 2.2$ & & 31.1 \\
\hline \multirow[t]{4}{*}{ AMT } & WT & + & $8.9 \pm 0.4$ & $38.9 \pm 0.1$ & 26.1 \\
\hline & & - & $17.9 \pm 2.1$ & $24.3 \pm 0.7$ & 28.9 \\
\hline & $\Delta 60$ & + & $21.5 \pm 1.9$ & $24.9 \pm 0.7$ & 26.8 \\
\hline & & - & $24.5 \pm 0.5$ & $18.1 \pm 1.6$ & 28.7 \\
\hline \multirow[t]{4}{*}{ IMP } & WT & + & $2.6 \pm 0.4$ & $49.7 \pm 2.0$ & 23.9 \\
\hline & & - & $6.5 \pm 1.6$ & $36.3 \pm 4.4$ & 28.6 \\
\hline & $\Delta 60$ & + & $15.8 \pm 0.6$ & $40.7 \pm 0.3$ & 21.7 \\
\hline & & - & $18.5 \pm 0.5$ & $33.1 \pm 0.6$ & 24.2 \\
\hline \multirow[t]{4}{*}{ OPZ } & WT & + & $21.7 \pm 0.8$ & $15.3 \pm 2.4$ & 31.5 \\
\hline & & - & $25.6 \pm 0.4$ & $6.8 \pm 0.1$ & 33.8 \\
\hline & $\Delta 60$ & + & $39.9 \pm 0.8$ & 0 & 30.1 \\
\hline & & - & $42.7 \pm 2.7$ & 0 & 28.7 \\
\hline \multirow[t]{4}{*}{ LPZ } & WT & + & $18.4 \pm 1.0$ & $6.5 \pm 2.1$ & 37.5 \\
\hline & & - & $24.9 \pm 0.6$ & $4.0 \pm 0.2$ & 35.5 \\
\hline & $\Delta 60$ & + & $45.5 \pm 0.7$ & 0 & 27.2 \\
\hline & & - & $48.9 \pm 1.3$ & 0 & 25.6 \\
\hline
\end{tabular}

Absorbance at $340 \mathrm{~nm}$ was measured following the complete consumption of $100 \mu \mathrm{M}$ NADPH, and the amounts of metabolites and $\mathrm{H}_{2} \mathrm{O}_{2}$ were determined. The amounts of $\mathrm{H}_{2} \mathrm{O}$ produced by shunt path 3 were calculated with Eq. 2 .

reported to promote formation of the oxygenated form. ${ }^{53,54)}$ Structural perturbation will be induced by binding with the redox partner, and may alter the electron flow from the thiolate ligand to the heme iron. ${ }^{55)}$

One role of the CPR membrane anchor is to allow CPR to move two-dimensionally on the membrane, and to orient CPR suitably to facilitate electron transfer to CYP. In this work, we constructed a truncated $\triangle 60-\mathrm{CPR}$ mutant and studied its binding affinity, electron transfer activity, and drug metabolizing property, using CYP2C19 as a redox partner. We demonstrated that $\triangle 60-C P R$ could metabolize AMT and IMP, and that defective metabolism of OPZ and LPZ could be attributed to the shunt path, possibly by disruption of the hydrogen bond network, depending on the drug type. Thus, the membrane anchor of human CPR plays a key role not only in mooring CPR to the membrane, but also in suppressing the shunt path and 
ensure efficient use of reducing equivalents.

Acknowledgments We thank Dr. Noritaka Ariyoshi in Chiba University Hospital for the generous gift of the human CPR gene. This work was financially supported by Grantsin-Aid for Scientific Research (23390011 to TU) from JSPS, Japan.

Conflict of Interest The authors declare no conflict of interest.

\section{References}

1) Shen A. L., O'Leary K. A., Kasper C. B., J. Biol. Chem., 277, 6536-6541 (2002).

2) Schacter B. A., Nelson E. B., Marver H. S., Masters B. S. S., J. Biol. Chem., 247, 3601-3607 (1972).

3) Ilan Z., Ilan R., Cinti D. L., J. Biol. Chem., 256, 10066-10072 (1981).

4) Enoch H. G., Strittmatter P., J. Biol. Chem., 254, 8976-8981 (1979).

5) Ono T., Bloch K., J. Biol. Chem., 250, 1571-1579 (1975).

6) Evans W. E., Relling M. V., Science, 286, 487-491 (1999).

7) Zanger U. M., Schwab M., Pharmacol. Ther., 138, 103-141 (2013).

8) Porter T. D., Kasper C. B., Proc. Natl. Acad. Sci. U.S.A., 82, 973 977 (1985).

9) Haniu M., Iyanagi T., Miller P., Lee T. D., Shively J. E., Biochemistry, 25, 7906-7911 (1986).

10) Yamano S., Aoyama T., McBride O. W., Hardwick J. P., Gelboin H. V., Gonzalez F. J., Mol. Pharmacol., 36, 83-88 (1989).

11) Wang M., Roberts D. L., Paschke R., Shea T. M., Masters B. S., Kim J. J., Proc. Natl. Acad. Sci. U.S.A., 94, 8411-8416 (1997).

12) Xia C., Panda S. P., Marohnic C. C., Martasek P., Masters B. S., Kim J. J., Proc. Natl. Acad. Sci. U.S.A., 108, 13486-13491 (2011).

13) Bernhardt R., Pommerening K., Ruckpaul K., Biochem. Int., 14, 823-832 (1987).

14) Bernhardt R., Kraft R., Otto A., Ruckpaul K., Biomed. Biochim. Acta, 47, 581-592 (1988).

15) Nadler S. G., Strobel H. W., Arch. Biochem. Biophys., 290, 277-284 (1991).

16) Shen A. L., Kasper C. B., J. Biol. Chem., 270, $27475-27480$ (1995).

17) Hasemann C. A., Kurumbail R. G., Boddupalli S. S., Peterson J. A., Deisenhofer J., Structure, 3, 41-62 (1995).

18) Bridges A., Gruenke L., Chang Y. T., Vakser I. A., Loew G., Waskell L., J. Biol. Chem., 273, 17036-17049 (1998).

19) Hamdane D., Xia C., Im S. C., Zhang H., Kim J. J., Waskell L., J. Biol. Chem., 284, 11374-11384 (2009).

20) Ellis J., Gutierrez A., Barsukov I. L., Huang W. C., Grossmann J. G., Roberts G. C., J. Biol. Chem., 284, 36628-36637 (2009).

21) Huang W. C., Ellis J., Moody P. C. E., Raven E. L., Roberts G. C. K., Structure, 21, 1581-1589 (2013).

22) Sündermann A., Oostenbrink C., Protein Sci., 22, 1183-1195 (2013).

23) Gum J. R., Strobel H. W., J. Biol. Chem., 256, 7478-7486 (1981).

24) Gideon D. A., Kumari R., Lynn A. M., Manoj K. M., Cell Biochem. Biophys., 63, 35-45 (2012).

25) Huang R., Yamamoto K., Zhang M., Popovych N., Hung I., Im S. C., Gan Z., Waskell L., Ramamoorthy A., Biophys. J., 106, 21262133 (2014)

26) Ingelman-Sundberg M., Biochim. Biophys. Acta, 488, 225-234 (1977).

27) Inui H., Maeda A., Ohkawa H., Biochemistry, 46, 10213-10221
(2007).

28) Lamb D. C., Warrilow A. G., Venkateswarlu K., Kelly D. E., Kelly S. L., Biochem. Biophys. Res. Commun., 286, 48-54 (2001).

29) Marohnic C. C., Panda S. P., Martasek P., Masters B. S., J. Biol. Chem., 281, 35975-35982 (2006).

30) Vermilion J. L., Coon M. J., J. Biol. Chem., 253, 2694-2704 (1978).

31) Black S. D., French J. S., Williams C. H. Jr., Coon M. J., Biochem. Biophys. Res. Commun., 91, 1528-1535 (1979).

32) Venkateswarlu K., Lamb D. C., Kelly D. E., Manning N. J., Kelly S. L., J. Biol. Chem., 273, 4492-4496 (1998).

33) Estabrook R. W., Shet M. S., Fisher C. W., Jenkins C. M., Waterman M. R., Arch. Biochem. Biophys., 333, 308-315 (1996).

34) Gilep A. A., Guryev O. L., Usanov S. A., Estabrook R. W., Biochem. Biophys. Res. Commun., 284, 937-941 (2001).

35) Attia T. Z., Yamashita T., Miyamoto M., Koizumi A., Yasuhara Y., Node J., Erikawa Y., Komiyama Y., Horii C., Yamada M., Omar M. A., Abdelmageed O. H., Derayea S. M., Uno T., Chem. Pharm. Bull., 60, 1544-1549 (2012).

36) Attia T. Z., Yamashita T., Hammad M. A., Hayasaki A., Sato T., Miyamoto M., Yasuhara Y., Nakamura T., Kagawa Y., Tsujino H., Omar M. A., Abdelmageed O. H., Derayea S. M., Uno T., Chem. Pharm. Bull., 62, 176-181 (2014).

37) Yasukochi Y., Masters B. S., J. Biol. Chem., 251, 5337-5344 (1976).

38) Oprian D. D., Coon M. J., J. Biol. Chem., 257, 8935-8944 (1982).

39) Guengerich F. P., Martin M. V., Sohl C. D., Cheng Q., Nat. Protoc., 4, 1245-1251 (2009).

40) Xia C., Hamdane D., Shen A. L., Choi V., Kasper C. B., Pearl N. M., Zhang H., Im S. C., Waskell L., Kim J. J., J. Biol. Chem., 286, 16246-16260 (2011).

41) Fang X., Kobayashi Y., Halpert J. R., FEBS Lett., 416, 77-80 (1997).

42) Hutzler J. M., Wienkers L. C., Wahlstrom J. L., Carlson T. J., Tracy T. S., Arch. Biochem. Biophys., 410, 16-24 (2003).

43) Wei L., Locuson C. W., Tracy T. S., Mol. Pharmacol., 72, 1280 1288 (2007).

44) Iwata H., Fujita K., Kushida H., Suzuki A., Konno Y., Nakamura K., Fujino A., Kamataki T., Biochem. Pharmacol., 55, 1315-1325 (1998).

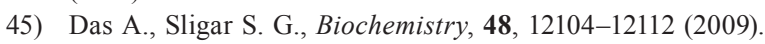

46) Khatri Y., Gregory M. C., Grinkova Y. V., Denisov I. G., Sligar S. G., Biochem. Biophys. Res. Commun., 443, 179-184 (2014).

47) Gorsky L. D., Koop D. R., Coon M. J., J. Biol. Chem., 259, 6812 6817 (1984).

48) Atkins W. M., Sligar S. G., Biochemistry, 27, 1610-1616 (1988).

49) Loida P. J., Sligar S. G., Biochemistry, 32, 11530-11538 (1993).

50) Zhao Y., White M. A., Muralidhara B. K., Sun L., Halpert J. R., Stout C. D., J. Biol. Chem., 281, 5973-5981 (2005).

51) Imai M., Shimada H., Watanabe Y., Matsushima-Hibiya Y., Makino R., Koga H., Horiuchi T., Ishimura Y., Proc. Natl. Acad. Sci. U.S.A., 86, 7823-7827 (1989).

52) Reynald R. L., Sansen S., Stout C. D., Johnson E. F., J. Biol. Chem., 287, 44581-44591 (2012).

53) Adachi S., Nagano S., Ishimori K., Watanabe Y., Morishima I., Egawa T., Kitagawa T., Makino R., Biochemistry, 32, 241-252 (1993).

54) Auclair K., Moënne-Loccoz P., Ortiz de Montellano P. R., J. Am. Chem. Soc., 123, 4877-4885 (2001).

55) Sjodin T., Christian J. F., Macdonald I. D., Davydov R., Unno M., Sligar S. G., Hoffman B. M., Champion P. M., Biochemistry, 40, 6852-6859 (2001). 